\title{
The Conceptual Framework of Quantum Field Theory
}

\author{
Anthony Duncan
}




\section{Contents}

1 Origins I: From the arrow of time to the first quantum field 1

$\begin{array}{ll}1.1 \text { Quantum prehistory: crises in classical physics } & 1\end{array}$

$\begin{array}{lll}1.2 & \text { Early work on cavity radiation } & 3\end{array}$

$\begin{array}{lll}1.3 & \text { Planck's route to the quantization of energy } & 8\end{array}$

$\begin{array}{lll}1.4 & \text { First inklings of field quantization: Einstein and energy fluctuations } & 14\end{array}$

1.5 The first true quantum field: Jordan and energy fluctuations 18

2 Origins II: Gestation and birth of interacting field theory:
from Dirac to Shelter Island

2.1 Introducing interactions: Dirac and the beginnings of quantum electrodynamics

2.2 Completing the formalism for free fields: Jordan, Klein, Wigner, Pauli, and Heisenberg

2.3 Problems with interacting fields: infinite seas, divergent integrals, and renormalization

3 Dynamics I: The physical ingredients of quantum field theory: $\begin{array}{ll}\text { dynamics, symmetries, scales } & 57\end{array}$

4 Dynamics II: Quantum mechanical preliminaries 69

$\begin{array}{lll}\text { 4.1 The canonical (operator) framework } & 70\end{array}$

4.2 The functional (path-integral) framework 86

$\begin{array}{lrr}4.3 & \text { Scattering theory } & 96\end{array}$

$\begin{array}{lll}4.4 \text { Problems } & 106\end{array}$

5 Dynamics III: Relativistic quantum mechanics 108

5.1 The Lorentz and Poincaré groups 108

5.2 Relativistic multi-particle states (without spin) 111

$\begin{array}{lll}5.3 & \text { Relativistic multi-particle states (general spin) } & 114\end{array}$

5.4 How not to construct a relativistic quantum theory 121

5.5 A simple condition for Lorentz-invariant scattering 125

$\begin{array}{lll}5.6 & \text { Problems } & 130\end{array}$

6 Dynamics IV: Aspects of locality: clustering, microcausality,

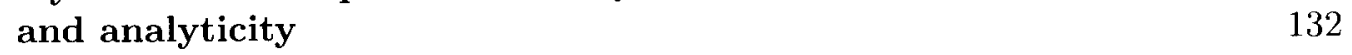

6.1 Clustering and the smoothness of scattering amplitudes 133

$\begin{array}{lll}6.2 & \text { Hamiltonians leading to clustering theories } & 138\end{array}$

6.3 Constructing clustering Hamiltonians: second quantization 144

$\begin{array}{ll}6.4 \text { Constructing a relativistic, clustering theory } & 149\end{array}$

6.5 Local fields, non-localizable particles! 159 
viii Contents

6.6 From microcausality to analyticity 164

$\begin{array}{ll}6.7 \text { Problems } & 169\end{array}$

7 Dynamics V: Construction of local covariant fields 171

7.1 Constructing local, Lorentz-invariant Hamiltonians 171

7.2 Finite-dimensional representations of the homogeneous 173

Lorentz group 173

7.3 Local covariant fields for massive particles of any spin:
the Spin-Statistics theorem

$\begin{array}{ll}7.4 & \text { Local covariant fields for spin- } \frac{1}{2} \text { (spinor fields) } \\ 7.54\end{array}$

7.5 Local covariant fields for spin-1 (vector fields) 198

$\begin{array}{ll}7.6 & \text { Some simple theories and processes } \\ 7.7 & 202\end{array}$

$\begin{array}{lll}7.7 & \text { Problems } & 215\end{array}$

8 Dynamics VI: The classical limit of quantum fields 219

$\begin{array}{ll}\text { 8.1 Complementarity issues for quantum fields } & 219\end{array}$

8.2 When is a quantum field "classical"? 223

$\begin{array}{ll}8.3 \text { Coherent states of a quantum field } & 228\end{array}$

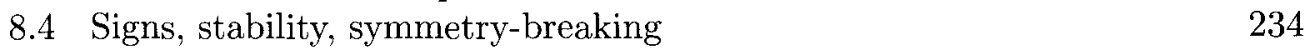

$\begin{array}{ll}8.5 \text { Problems } & 238\end{array}$

9 Dynamics VII: Interacting fields: general aspects 240

9.1 Field theory in Heisenberg representation: heuristics 241

9.2 Field theory in Heisenberg representation: axiomatics 253

9.3 Asymptotic formalism I: the Haag-Ruelle scattering theory 268

9.4 Asymptotic formalism II: the Lehmann-Symanzik-Zimmermann 281

9.5 Spectral properties of field theory 289

9.6 General aspects of the particle-field connection 297

$\begin{array}{lll}9.7 \text { Problems } & 304\end{array}$

10 Dynamics VIII: Interacting fields: perturbative aspects 307

10.1 Perturbation theory in interaction picture and Wick's theorem

10.2 Feynman graphs and Feynman rules 314

$\begin{array}{lll}10.3 & \text { Path-integral formulation of field theory } & 325\end{array}$

10.4 Graphical concepts: $N$-particle irreducibility 341

10.5 How to stop worrying about Haag's theorem 359

$\begin{array}{lll}10.6 & \text { Problems } & 371\end{array}$

11 Dynamics IX: Interacting fields: non-perturbative aspects 374

$\begin{array}{ll}11.1 & \text { On the (non-) convergence of perturbation theory } \\ 11.2 & 376\end{array}$

11.2 "Perturbatively non-perturbative" processes: threshhold bound states

11.3 "Essentially non-perturbative" processes: non-Borel-summability in field theory

11.4 Problems 
12 Symmetries I: Continuous spacetime symmetry: why we need Lagrangians in field theory

12.1 The problem with derivatively coupled theories: seagulls, Schwinger terms, and $T^{*}$ products

12.2 Canonical formalism in quantum field theory

12.3 General condition for Lorentz-invariant field theory

12.4 Noether's theorem, the stress-energy tensor, and all that stuff

12.5 Applications of Noether's theorem

12.6 Beyond Poincaré: supersymmetry and superfields

12.7 Problems

13 Symmetries II: Discrete spacetime symmetries 469

13.1 Parity properties of a general local covariant field 470

13.2 Charge-conjugation properties of a general local covariant field 474

13.3 Time-reversal properties of a general local covariant field 477

13.4 The TCP and Spin-Statistics theorems 478

13.5 Problems 485

14 Symmetries III: Global symmetries in field theory 487

14.1 Exact global symmetries are rare! 489

14.2 Spontaneous breaking of global symmetries: the Goldstone theorem 492

14.3 Spontaneous breaking of global symmetries: dynamical aspects 495

$\begin{array}{lll}14.4 & \text { Problems } & 507\end{array}$

15 Symmetries IV: Local symmetries in field theory 509

15.1 Gauge symmetry: an example in particle mechanics 509

15.2 Constrained Hamiltonian systems 512

15.3 Abelian gauge theory as a constrained Hamiltonian system 519

15.4 Non-abelian gauge theory: construction and functional integral formulation 529

15.5 Explicit quantum-breaking of global symmetries: anomalies 544

15.6 Spontaneous symmetry-breaking in theories with a local gauge symmetry 552

15.7 Problems $\quad 565$

Scales I: Scale sensitivity of field theory amplitudes and
effective field theories

16.1 Scale separation as a precondition for theoretical science $\quad 570$

16.2 General structure of local effective Lagrangians 571

16.3 Scaling properties of effective Lagrangians: relevant, marginal,
and irrelevant operators

16.4 The renormalization group $\quad 581$

16.5 Regularization methods in field theory 588

16.6 Effective field theories: a compendium 595

$\begin{array}{lll}16.7 & \text { Problems } & 608\end{array}$ 
X Contents

17 Scales II: Perturbatively renormalizable field theories

17.1 Weinberg's power-counting theorem and the divergence structure of Feynman integrals

17.2 Counterterms, subtractions, and perturbative renormalizability

17.3 Renormalization and symmetry

17.4 Renormalization group approach to renormalizability

17.5 Problems

18 Scales III: Short-distance structure of quantum field theory

18.1 Local composite operators in field theory

18.2 Factorizable structure of field theory amplitudes: the operator product expansion

18.3 Renormalization group equations for renormalized amplitudes

18.4 Problems

19 Scales IV: Long-distance structure of quantum field theory

19.1 The infrared catastrophe in unbroken abelian gauge theory

19.2 The Bloch-Nordsieck resolution

724

19.3 Unbroken non-abelian gauge theory: confinement

728

19.4 How confinement works: three-dimensional gauge theory

19.5 Problems

Appendix A The functional calculus

Appendix B Rates and cross-sections

Appendix C Majorana spinor algebra 